\title{
NEAR-INFRARED SPECTROSCOPY OF PROTO-PLANETARY NEBULAE
}

\section{B.J. HRIVNAK}

Dept. of Physics $\& 3$ Astronomy, Valparaiso University, Valparaiso, IN 46383, USA

S. KWOK

Dept. of Physics $\&$ Astronomy, University of Calgary, Calgary, AB T2N 1N4, CANADA

and

T.R. GEBALLE

Joint Astronomy Center, 665 Komohana St., Hilo, HI 96720, USA

Sixteen candidates for proto-planetary nebulae have been observed with lowresolution infrared spectroscopy in the $\mathrm{H}$ and $\mathrm{K}$ bands, and 6 in the $\mathrm{L}$ band, using the United Kingdom Infrared Telescope. In the $\mathrm{H}$ band, the objects show hydrogen Brackett lines in absorption. In the $\mathrm{K}$ band, absorption bands (del $\mathrm{v}=2$ ) of $\mathrm{CO}$ were observed, and in three cases the $\mathrm{CO}$ bands are in emission. The $\mathrm{CO}$ spectrum of IRAS $22272+5435$ was found to change from emission to absorption over a threemonth interval. This $\mathrm{CO}$ emission can be interpreted as an indication of some recent episodes of mass loss in these objects. Four of the objects were found to possess an emission feature at $3.3 \mathrm{um}$, usually associated with PAHs, and two of these show an unusually strong $3.4 \mathrm{um}$ emission feature (Geballe, Tielens, Kwok, \& Hrivnak 1992, ApJ, 387, L89). 